\title{
Agroinfiltration of Cauliflower mosaic virus Gene VI Elicits Hypersensitive Response in Nicotiana Species
}

\author{
Karuppaiah Palanichelvam, Anthony B. Cole, Monir Shababi, and James E. Schoelz \\ Department of Plant Microbiology and Pathology, University of Missouri, Columbia 65211, U.S.A. \\ Accepted 8 August 2000.
}

Cauliflower mosaic virus strain W260 induces hypersensitive response (HR) in Nicotiana edwardsonii and systemic cell death in $N$. clevelandii. In contrast, the D4 strain of Cauliflower mosaic virus evades the host defenses in Nicotiana species; it induces chlorotic primary lesions and a systemic mosaic in both hosts. Previous studies with chimeric viruses had indicated that gene VI of W260 was responsible for elicitation of $\mathrm{HR}$ or cell death. To prove conclusively that $\mathrm{W} 260$ gene $\mathrm{VI}$ is responsible, we inserted gene VI of W260 and D4 into the Agrobacterium tumefaciens binary vector pKYLX7. Agroinfiltration of these constructs into the leaves of $N$. edwardsonii and $N$. clevelandii revealed that gene VI of $\mathrm{W} 260$ elicited HR in $N$. edwardsonii 4 to 5 days after infiltration and cell death in $N$. clevelandii approximately 9 to 12 days after infiltration. In contrast, gene VI of D4 did not elicit HR or cell death in either Nicotiana species. A frameshift mutation introduced into gene VI of W260 abolished its ability to elicit HR or cell death in both Nicotiana species, demonstrating that the elicitor is the gene VI protein.

The hypersensitive response (HR) is a plant defense response characterized by localized cell death around the initial point of pathogen attack and by the production of proteins and chemicals that inhibit the spread of the pathogen in the host (Staskawicz et al. 1995). HR is triggered when a host resistance $(R)$ gene product in the plant recognizes a pathogen avirulence ( $a v r$ ) gene product. Avr genes have been characterized for many different plant pathogens. Recently it has been shown that avr genes are able to elicit HR in heterologous systems. We have found that Cauliflower mosaic virus (CaMV) strain W260 can trigger a hypersensitive response in Nicotiana edwardsonii and a systemic cell death symptom in $N$. clevelandii (Király et al. 1999). In contrast to W260, CaMV strain D4 is apparently able to evade the plant defense response induced in N. edwardsonii and does not elicit the cell death symptom in $N$. clevelandii. The D4 strain of CaMV induces a systemic mosaic in both hosts. Studies with chimeric viruses constructed between D4 and W260 have indicated that CaMV gene VI should be considered an avr gene. The W260 gene VI appears to contain the sequence elements

Corresponding author: J. Schoelz; Telephone: +1-573-882-1185; Fax: +1-573-882-0588; E-mail: schoelzj@missouri.edu for elicitation of HR in N. edwardsonii and is responsible for the formation of a systemic cell death symptom in $N$. clevelandii (Király et al. 1999).

At least two problems have limited the acceptance of CaMV gene VI as a legitimate $a v r$ gene. The first problem is related to the function of gene VI in the viral infection cycle. There is extensive evidence that gene VI is required for efficient translation of the other viral genes, genes $\mathrm{I}$ to $\mathrm{V}$ (Bonneville et al. 1989; Fütterer and Hohn 1991; Gowda et al. 1989). Thus, there is a possibility that gene VI could influence the host range indirectly through modulating the expression of one of the other viral gene products. Second, it has been shown that other viral gene products, in particular the aphid transmission factor (gene II) and the viral capsid protein (gene IV), contribute to the ability of some CaMV strains to infect some Nicotiana species (Qiu and Schoelz 1992). Consequently, it has been considered that complex interactions govern the ability of CaMV to infect Nicotiana species systemically rather than a simple gene-for-gene interaction (Dawson and Hilf 1992).

To conclusively determine whether gene VI is an avr gene, we have sought to express this protein through the technique of agroinfiltration. The rationale is that if W260 gene VI is responsible for elicitation of HR in N. edwardsonii or cell death in $N$. clevelandii, then it should be able to do so when expressed separately from the remainder of the viral genome. Agroinfiltration has been used successfully to illustrate the function of avr genes from several organisms, including Tobacco mosaic virus (Abbink et al. 1998; Erickson et al. 1999), Potato virus X (Bendahmane et al. 1999), Cladosporium fulvum (Thomas et al. 2000; Van der Hoorn et al. 2000), Pseudomonas syringae pv. tomato (Scofield et. al. 1996; Tang et al. 1996), and Xanthomonas campestris pv. vesicatoria (Van den Ackerveken et al. 1996).

Oligonucleotide primers were designed to amplify gene VI of W260 and D4 such that an XhoI site was present immediately upstream from the initiation codon and a KpnI site was immediately downstream from the stop codon. The gene VI coding regions of D4 and W260 were amplified from infectious clones of these viruses, and the amplified sequences were inserted into pGEM-7Zf(-) (Promega, Madison, WI, U.S.A.). To verify that the polymerase chain reaction process did not introduce any errors into the sequence, the nucleotide sequences of the amplified DNAs were determined and clones that matched the sequence of the original virus strains were 
selected. The gene VI coding regions of D4 and W260 were subsequently cloned between the border regions of the Agrobacterium tumefaciens binary vector pKYLX7 (Schardl et al. 1987). As shown in Figure 1A, gene VI of W260 and D4 were placed under the control of the $35 \mathrm{~S}$ promoter and rbcs terminator to make the constructs pW260VI and pD4VI, respectively. The constructs were then mobilized into A. tumefaciens strain C58 (GV2260) by triparental mating using the mobilizing functions of pRK2013 (Ditta et al. 1990). In the transient expression assays, A. tumefaciens cultures were preinduced overnight with $100 \mu \mathrm{m}$ of acetosyringone and infiltrated at a cell density of 1.0 optical density $(600 \mathrm{~nm})$, as described by Van der Hoorn et al. (2000).

To confirm that gene VI was expressed from our constructs, total RNA was isolated from leaf panels that had been infiltrated with pW260VI or pD4VI. The RNA was separated on a denaturing agarose gel and transferred to nylon membranes,
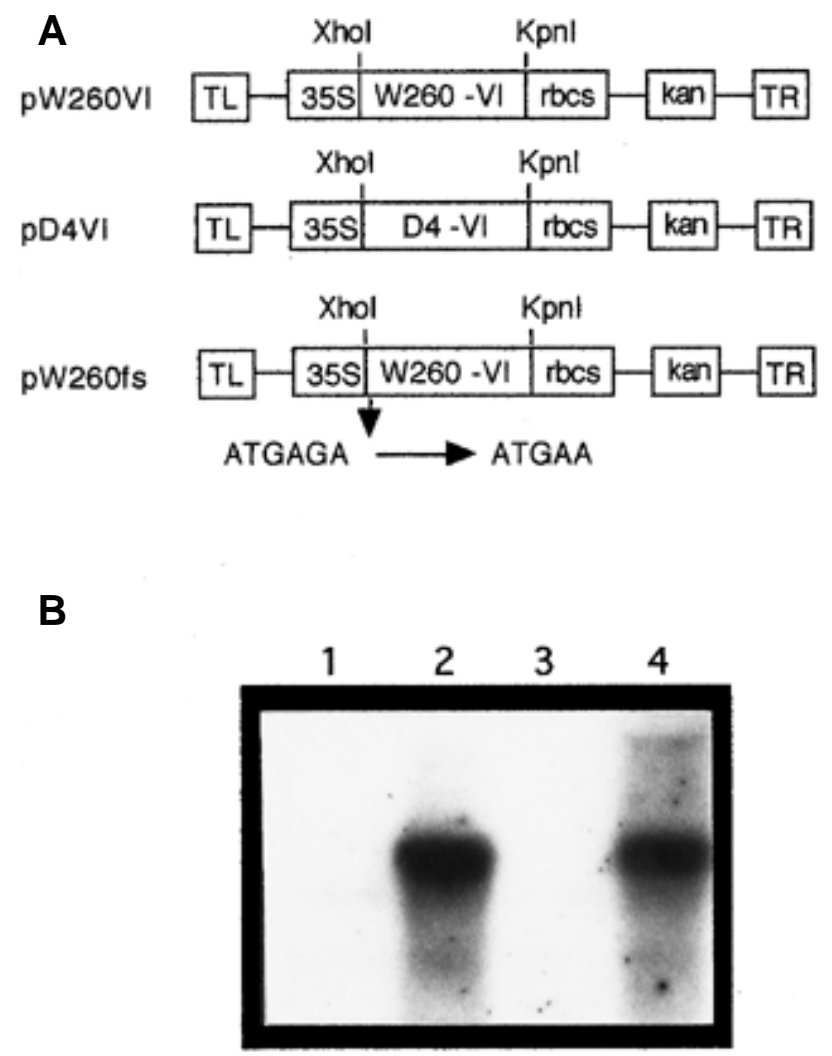

Fig. 1. Schematic representation of gene VI constructs used for agroinfiltration into leaf panels. A, The polymerase chain reaction-amplified gene VI coding regions of W260 and D4 were cloned into the Agrobacterium tumefaciens binary vector pKYLX7 to create plasmids pW260VI and pD4VI, respectively. In plasmid pW260fs, a frameshift mutation was introduced into the second codon of W260 gene VI. Expression of gene VI was under the control of the Cauliflower mosaic virus $35 \mathrm{~S}$ promoter and rbcs terminator. The TL and TR sequences correspond to the left and right border of the T-DNA sequence in the vector. B, A Northern blot of total leaf RNA after hybridization with ${ }^{32} \mathrm{P}$-labeled gene VI DNA. Total RNA was extracted at 4 days postinfiltration. RNA $(10 \mu \mathrm{g})$ was loaded into each well and blotted onto a nylon membrane after electrophoresis. The amount of RNA was verified by staining with ethidium bromide in a duplicate agarose gel. Lane 1, noninfiltrated leaf tissue; lane 2, leaf tissue infiltrated with pW260VI; lane 3, leaf tissue infiltrated with the empty vector pKYLX7; lane 4, leaf tissue infiltrated with pD4VI. which were subsequently probed with ${ }^{32} \mathrm{P}$-labeled gene VI DNA. Transcripts were detected only in leaf panels infiltrated with A. tumefaciens containing pW260VI or pD4VI (Fig. 1B). A comparison of the intensity of signals from pW260VI and pD4VI suggested that the level of expression of gene VI of both strains was approximately the same. No signal was detected in panels infiltrated with the empty vector, pKYLX7, or a buffer control. In addition, the plasmids pW260VI and pD4VI were used for stable transformation of Arabidopsis thaliana, and a Western blot confirmed that the W260 and D4 P6 proteins were expressed in the transformed plants (W. Yu and J. Schoelz, unpublished).

To determine whether gene VI of W260 has characteristics of an $a v r$ gene, pW260VI and pD4VI were agroinfiltrated into $N$. edwardsonii, $N$. edwardsonii cv. Columbia, and N. clevelandii, Nicotiana species that respond to W260 virions with HR or systemic cell death (Table 1). Columbia differs from N. edwardsonii in that Columbia has the full complement of chromosomes from $N$. glutinosa and $N$. clevelandii, whereas $N$. edwardsonii lacks two pairs of chromosomes (A. Cole and J. Schoelz, unpublished). Both types of plants, however, respond to W260 infection with HR. In the agroinfiltration assay, gene VI of W260 triggered HR in the two N. edwardsonii cultivars 4 to 6 days postinfiltration (dpi) (Fig. 2A) and cell death in N. clevelandii at 9 to 12 dpi (Fig. 2B). To confirm that HR or cell death was not triggered by A.tumefaciens, we found that $A$. tumefaciens carrying the empty vector pKYLX7 would not elicit HR or cell death in N. edwardsonii or N. clevelandii, respectively (Fig. $2 \mathrm{~A}$ and $\mathrm{B})$. To support the data that the induction of necrosis observed in Nicotiana spp. was caused by A. tumefaciensmediated expression, the same binary vector constructs were agroinfiltrated without the vir helper plasmid pGV2260. No necrosis was elicited by A. tumefaciens strains lacking the vir helper plasmid, an indication that T-DNA transfer was required for induction of HR (data not shown). These experiments show that gene VI of W260 is sufficient to elicit HR in N. edwardsonii and cell death in $N$. clevelandii. Significantly, gene VI of D4 did not induce cell death in any of the Nicotiana species tested (Fig. 2A and B). This is consistent with the observation that the D4 virus does not induce local or systemic cell death in $N$. edwardsonii and $N$. clevelandii; rather, this virus causes a systemic mosaic in both hosts.

Although evidence has existed since 1984 that CaMV gene VI can function as an $a v r$ gene (Daubert et al. 1984), it has not been possible to prove conclusively that induction of HR is dependent upon the expression of the gene VI protein product.

Fig. 2. The effect of gene VI of strains W260 and D4 after Agrobacterium tumefaciens-mediated T-DNA transfer into Nicotiana spp. A, $N$. edwardsonii cv. Columbia at 9 dpi (days postinfiltration); B, N. clevelandii at $14 \mathrm{dpi}$; C, N. edwardsonii at $8 \mathrm{dpi}$; D, N. bigelovii at $18 \mathrm{dpi} .1$, Infiltration with buffer control; 2 , infiltration with $A$. tumefaciens harboring the empty vector pKYLX7; 3, infiltration with A. tumefaciens harboring pD4VI; 4, infiltration with A. tumefaciens harboring pW260VI. 5, coinfiltration with A. tumefaciens strains harboring pW260VI and pD4VI mixed in a 1:1 ratio. In a typical experiment, each construct was infiltrated into two fully expanded leaves on two separate plants (four leaves per experiment). Plants varied in age from 45 to 90 days postplanting. To compare constructs within an experiment, each construct was infiltrated onto the same leaf. The number of experiments for each Nicotiana species varied between 8 (N. bigelovii) and $11(\mathrm{~N}$. edwardsonii and Columbia), with similar results in each experiment. 

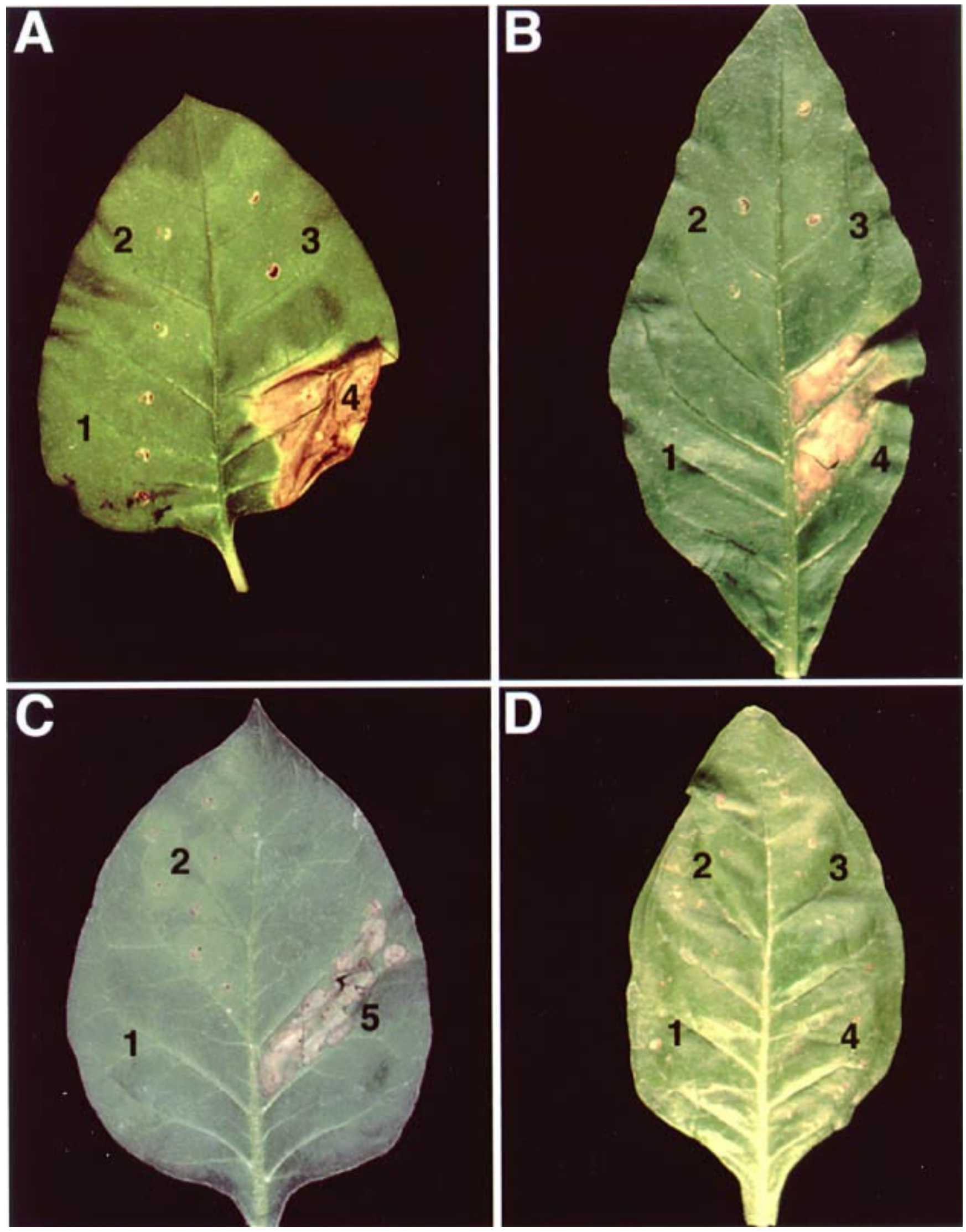

Vol. 13 , No. $11,2000 / 1277$ 
To determine whether elicitation of HR was mediated by the gene VI mRNA or the protein product, a deletion of a single base was introduced into the second codon after the gene VI start codon. This frameshift mutant, pW260fs (Fig. 1A), was unable to elicit HR upon agroinfiltration into N. edwardsonii. In a parallel set of experiments, we found that pW260fs was unable to elicit cell death in $N$. clevelandii (data not shown). We concluded that elicitation of HR or cell death was dependent on the expression of the W260 gene VI protein product.

To investigate whether P6 of D4 could suppress the development of HR induced by W260 P6, A. tumefaciens strains harboring pW260VI and pD4VI were mixed in a $1: 1$ ratio and infiltrated into leaf panels of $N$. edwardsonii. HR developed in these leaves at the same rate as in N. edwardsonii leaves infiltrated with only pW260VI, indicating that gene VI of D4 could not suppress the activity of gene VI of W260 (Fig. 2C). This result is consistent with the observation that the W260 virus actively elicited host defenses (i.e., HR and, subsequently, pathogenesis-related proteins) in N. edwardsonii, whereas the D4 virus did not elicit host defenses (Cole et al. 1999). Taken together, the two studies indicate that gene VI of D4 may allow the virus to evade host defenses rather than actively suppressing a host defense response.

In contrast to $N$. edwardsonii and $N$. clevelandii, $N$. bigelovii develops a systemic mosaic symptom upon infection by CaMV strain W260. This host does not develop any cell death symptom in either inoculated or systemically infected leaves (Király et al. 1999). Genetic crosses between N. bigelovii and $N$. clevelandii have revealed that a single, dominant gene is responsible for cell death suppression (Király et al. 1999). We found that $N$. bigelovii was able to suppress the development of cell death induced by W260 gene VI in the agroinfiltration assay, but suppression was dependent on the age of the plants. $N$. bigelovii plants that were at least 75 days old were able to completely suppress the development of cell death induced by W260 gene VI. Leaves from these older plants were infiltrated with W260 gene VI and did not develop any symptoms of cell death during the 20-day period of the test (Fig. 2D). Interestingly, $N$. bigelovii plants that were 45 to 60 days old at the time of agroinfiltration of W60 gene VI were able to only delay the development of the cell death symptom. N. bigelovii leaf panels that had been agroinfiltrated with gene VI of W260 developed cell death at approximately 14 dpi (data not shown), whereas cell death appeared in the $N$. clevelandii leaf panels at 10 to $12 \mathrm{dpi}$.
The agroinfiltration studies confirmed that $N$. bigelovii can suppress the development of cell death induced by W260 gene VI, but apparently the suppressor is not as effective in young plants as it is in older plants. This age-dependent suppression might be explained by an increase in concentration of the suppressor as the plants grow older, but to fully understand how it functions, it will be necessary to identify and clone the cell death suppressor. In contrast to the agroinfiltration assay, even young $N$. bigelovii plants inoculated with W260 virions do not develop a systemic cell death symptom; these plants develop only a systemic mosaic (Király et al. 1999). The inability of young $N$. bigelovii plants to completely block the development of cell death in the agroinfiltration assay may also be a result of the artificial nature of the agroinfiltration assay. This assay may deliver a higher concentration of W260 P6 protein into a cell, overwhelming the cell death suppressor in these plants.

The gene VI product has been stably transformed into a number of plant species, and it has been found that expression of this transgene results in viruslike symptoms (Baughmann et al. 1988; Cecchini et al. 1997), including the induction of cell death (Goldberg et al. 1991; Takahashi et al. 1989). To determine whether the gene VI product acts as a nonspecific elicitor of cell death, we agroinfiltrated gene VI of W260 into the nonhost, $N$. tabacum, and observed plants for up to $20 \mathrm{dpi}$. The only symptom observed in all of the infiltrated leaf panels was a mild chlorosis similar to that illustrated in Figure 2A (position 2). This symptom was not associated with the infiltration of CaMV gene VI, as it also occurred in leaves that had been agroinfiltrated with the empty vector pKYLX7.

The evidence presented here indicates that the agroinfiltration of gene VI can provide an accurate assessment of the interaction between CaMV strains and Nicotiana species. W260 P6 was shown to elicit HR in both N. edwardsonii cultivars and cell death in $N$. clevelandii, and these reactions are in agreement with findings of studies involving wild-type and chimeric viruses (Table 1). The primary advantage of the agroinfiltration technique is that it induces a host response more rapid than that of virion inoculations (Table 1). We have purposely drawn a distinction between the HR induced by W260 P6 in N. edwardsonii and cell death induced by W260 P6 in N. clevelandii. The difference in terminology (HR versus cell death) reflects the fact that the W260 virus elicits a plant defense response in addition to cell death in N. edwardsonii, whereas W260 induces a systemic cell death symptom

Table 1. Comparison of host responses after Cauliflower mosaic virus (CaMV) virion inoculation or agroinfiltration of CaMV gene VI

\begin{tabular}{|c|c|c|c|c|}
\hline \multirow[b]{2}{*}{ Host } & \multicolumn{4}{|c|}{ Inoculum source } \\
\hline & W260 virions & $\begin{array}{l}\text { W260 gene VI } \\
\text { agroinfiltration }\end{array}$ & D4 virions & $\begin{array}{c}\text { D4 gene VI } \\
\text { agroinfiltration }\end{array}$ \\
\hline Nicotiana edwardsonii & $\mathrm{HR}^{\mathrm{a}}$ at 8 to $10 \mathrm{dpi}^{\mathrm{b}}$ & $\mathrm{HR}$ at $4 \mathrm{dpi}$ & Systemic mosaic $^{c}$ & No reaction \\
\hline N. edwardsonii cv. Columbia & $\mathrm{HR}$ at 8 to $10 \mathrm{dpi}$ & HR at 6 dpi & Systemic mosaic & No reaction \\
\hline N. clevelandii & Systemic cell death and mosaic & Cell death at 9 to $12 \mathrm{dpi}$ & Systemic mosaic & No reaction \\
\hline$N$. bigelovii, 2 months ${ }^{\mathrm{d}}$ & Systemic mosaic & Cell death at 14 dpi & Systemic mosaic & No reaction \\
\hline$N$. bigelovii, 3 months ${ }^{\mathrm{e}}$ & Systemic mosaic & No reaction & Systemic mosaic & No reaction \\
\hline N. tabacum & No infection & No reaction & No infection & No reaction \\
\hline
\end{tabular}

a Hypersensitive response.

b Days postinfiltration.

c In all cases, systemic mosaic was preceded by chlorotic local lesions at 8 to $10 \mathrm{dpi}$.

d Plants were agroinfiltrated at 2 months postplanting.

e Plants were agroinfiltrated at 3 months postplanting. 
in $N$. clevelandii (Table 1). The elicitation of host defenses in $N$. edwardsonii may contribute to the earlier onset of the cell death pathway by W260 P6 in that host relative to cell death induced in $N$. clevelandii (Table 1 ). We are in the process now of introgressing resistance from $N$. edwardsonii cv. Columbia into $N$. clevelandii to investigate whether the addition of this $\mathrm{CaMV}$ resistance gene can alter the timing of the cell death reaction induced during the agroinfiltration assay.

In contrast to P6 of W260, the D4 P6 did not elicit HR or cell death in any Nicotiana species we tested. A comparison of the primary sequences has shown that there are 34 amino acid differences between P6 of D4 and W260 (Daubert and Routh 1990; Wintermantel et al. 1993). Studies with chimeric viruses have further delineated the sequences that elicit $\mathrm{HR}$ in $N$. edwardsonii and cell death in $N$. clevelandii to the $\mathrm{N}$ terminal third of the protein, and most of the amino acid differences (21 of the 34) are found within this region (Király et al. 1999; Wintermantel et al. 1993). Daubert and Routh (1990) reported two mutations in the D4 gene VI sequence that influenced the development of HR in D. stramonium. Interestingly, neither of these mutations are present in the corresponding location of the W260 sequence, so more work needs to be done to characterize the "cell death" domain within CaMV gene VI. Agroinfiltration can provide a rapid method to screen site-directed mutants and also will be useful for evaluating the relationship between elicitation of cell death and translational transactivation, the primary function assigned to CaMV gene VI.

\section{ACKNOWLEDGMENTS}

The authors thank B. Baker for the gift of A. tumefaciens strain C58 (GV2260) and for helpful assistance with the agroinfiltration procedure. The authors also thank D. Pinkerton for assistance with the photographs. This research was supported by a grant from the Food for the 21 st Century program at the University of Missouri and by U.S. Department of Agriculture National Research Initiative Competitive Grant 98-353036711. This is a contribution from the Missouri Agricultural Experiment Station, Journal Series 13,060.

\section{LITERATURE CITED}

Abbink, T. E. M., Tjernberg, P. A., Bol, J. F., and Linthorst, H. J. M. 1998. Tobacco mosaic virus helicase domain induces necrosis in $N$ gene-carrying tobacco in the absence of virus replication. Mol. PlantMicrobe Interact. 11:1242-1246.

Baughmann, G. A., Jacobs, J. D., and Howell, S. H. 1988. Cauliflower mosaic virus gene VI produces a symptomatic phenotype in transgenic tobacco plants. Proc. Natl. Acad. Sci. USA 85:733-737.

Bendahmane, A., Kanyuka, K., and Baulcombe D. C. 1999. The $R x$ gene from potato controls separate virus resistance and cell death responses. Plant Cell 11:781-791.

Bonneville, J. M., Sanfacon, H., Fütterer, J., and Hohn, T. 1989. Posttranscriptional transactivation in cauliflower mosaic virus. Cell 59:1135-1143.

Cecchini, E., Gong, Z., Geri, C., Covey, S. N., and Milner, J. J. 1997. Transgenic Arabidopsis lines expressing gene VI protein from cauliflower mosaic virus variants exhibit a range of symptom-like phenotypes and accumulate inclusion bodies. Mol. Plant-Microbe Interact. 10:1094-1101.
Cole, A. B., Király, L., and Schoelz, J. E. 1999. Dissecting the hypersensitive response of Nicotiana edwardsonii to CaMV: Resistance and necrosis are determined by two separate plant genes. Phytopathology 89:S16.

Daubert, S., and Routh, G. 1990. Point mutations in cauliflower mosaic virus gene VI confer host-specific symptom changes. Mol. PlantMicrobe Interact. 3:341-345.

Daubert, S. D., Schoelz, J. E., Debao, L., and Shepherd, R. J. 1984. Expression of disease symptoms in cauliflower mosaic virus genomic hybrids. J. Mol. Appl. Genet. 2:537-547.

Dawson, W. O., and Hilf, M. E. 1992. Host-range determinants of plant viruses. Annu. Rev. Plant Physiol. Plant Mol. Biol. 43:527-555.

Ditta, G., Stanfield, S., Corbin, D., and Helinski, D. R. 1990. Broad host range DNA cloning system for Gram-negative bacteria: Construction of a gene bank of Rhizobium meliloti. Proc. Natl. Acad. Sci. USA 77:7347-7351.

Erickson, F., Holzberg, S., Calderon-Urrea, A., Handley, V., Axtell, M. Corr, C., and Baker, B. 1999. The helicase domain of the TMV replicase proteins induces the $N$-mediated defense response in tobacco. Plant J. 18:67-75

Fütterer, J., and Hohn, T. 1991. Translation of a polycistronic mRNA in the presence of the cauliflower mosaic virus transactivator protein. EMBO J. 10:3887-3896.

Goldberg, K. B., Kiernan, J., and Shepherd, R. J. 1991. A disease syndrome associated with expression of gene VI of caulimoviruses may be a nonhost reaction. Mol. Plant-Microbe Interact. 4:182-189.

Gowda, S., Wu, F. C., Scholthof, H. B., and Shepherd, R. J. 1989. Gene VI of figwort mosaic virus (caulimovirus group) functions in posttranscriptional expression of genes on the full-length RNA transcript. Proc. Natl. Acad. Sci. USA 86:9203-9207.

Király, L., Cole, A. B., Bourque, J. E., and Schoelz, J. E. 1999. Systemic cell death is elicited by the interaction of a single gene in Nicotiana clevelandii and gene VI of cauliflower mosaic virus. Mol. PlantMicrobe Interact. 12:919-925.

Qiu, S. G., and Schoelz, J. E. 1992. Three regions of cauliflower mosaic virus strain W260 are involved in systemic infection of solanaceous hosts. Virology 190:773-782.

Schardl, C. L., Byrd, A. D., Benzion, G., Altschuler, M. A., Hildebrand, D. F., and Hunt, A. G. 1987. Design and construction of a versatile system for the expression of foreign genes in plants. Gene 61:1-11.

Scofield, S. R., Tobias, C. M., Rathjen, J. P., Chang, J. H., Lavell, D. T., Michelmore, R. W., and Staskawicz, B. J. 1996. Molecular basis of gene-for-gene specificity in bacterial speck disease of tomato. Science 274:2063-2065.

Staskawicz, B. J., Ausubel, F. M., Baker, B. J., Ellis, J. G., and Jones, J. D. G. 1995. Molecular genetics of plant disease resistance. Science 268:661-666

Takahashi, H., Shimamoto, K., and Ehara, Y. 1989. Cauliflower mosaic virus gene VI causes growth suppression, development of necrotic spots and expression of defense related genes in transgenic tobacco plants. Mol. Gen. Genet. 216:188-194.

Tang, X., Frederick, R. D., Zhou, J., Halterman, D. A., Jia, Y., and Martin, G. B. 1996. Initiation of plant disease resistance by physical interaction of AvrPto and Pto kinase. Science 274:2060-2063.

Thomas, C. M., Tang, S., Hammond-Kosack, K., and Jones, J. D. G. 2000. Comparison of the hypersensitive response induced by the tomato $C f-4$ and $C f-9$ genes in Nicotiana spp. Mol. Plant-Microbe Interact. 13:465-469.

Van den Ackerveken, G., Marois, E., and Bonas, U. 1996. Recognition of the bacterial avirulence protein $A v r B s 3$ occurs inside the host plant cell. Cell 87:1307-1316.

Van der Hoorn, R. A. L., Laurent, F., Roth, R., and De Wit, P. J. G. M. 2000. Agroinfiltration is a versatile tool that facilitates comparative analyses of $A v r 9 / C f$-9-induced and $A v r 4 / C f$-4-induced necrosis. Mol. Plant-Microbe Interact. 13:439-446.

Wintermantel, W. M., Anderson, E. J., and Schoelz, J. E. 1993. Identification of domains within gene VI of cauliflower mosaic virus that influence systemic infection of Nicotiana bigelovii in a light-dependent manner. Virology 196:789-798. 